\title{
Antalya İli’nde Üretilen Gökkuşağı Alabalığı (Oncorhynchus mykiss, Walbaum 1792) Popülasyonundan Büyüme Hormonu I Geni İzolasyonu ve Karakterizasyonu
}

\author{
Mesut Yılmaz ${ }^{1 *}$, Mehmet Özbaş², Mehmet Akif Kılıç \\ 1* Akdeniz Üniversitesi, Su Ürünleri Fakültesi, Yetiştiricilik Bölümü, Antalya, Türkiye, (ORCID: 0000-0001-8799-3452), myilmaz@akdeniz.edu.tr \\ 2 Akdeniz Üniversitesi, Su Ürünleri Fakültesi, Yetiştiricilik Bölümü, Antalya, Türkiye, (ORCID: 0000-0001-6277-1095), mozbas@ akdeniz.edu.tr \\ ${ }^{3}$ Akdeniz Üniversitesi, Fen Fakültesi, Biyoloji Bölümü, Antalya, Türkiye, (ORCID: 0000-0002-4356-3343), mkilic@akdeniz.edu.tr
}

(İlk Geliş Tarihi 17 Ağustos 2020 ve Kabul Tarihi 8 Ekim 2020)

(DOI: $10.31590 /$ ejosat.781631)

ATIF/REFERENCE: Yılmaz, M., Özbaş, M., \& Kılıç, M.A. (2020). Antalya İli’nde Üretilen Gökkuşağı Alabalığı (Oncorhynchus mykiss, Walbaum 1792) Popülasyonundan Büyüme Hormonu I Geni İzolasyonu ve Karakterizasyonu. Avrupa Bilim ve Teknoloji Dergisi, (20), 223-232.

$\ddot{O} z$

Bu çalışmada, Antalya İli’nde yetiştirilen gökkuşağı alabalığı kültür popülasyonundan büyüme hormonu I geni izole ve karakterize edilmiştir. Bu amaçla kültür popülasyonundan 10 birey örnek olarak alınmıştır. Örneklenen balıkların kas dokularından toplam DNA izole edilmiştir. Polimeraz zincir reaksiyonunda kullanılmak üzere primerler dizayn edilmiş ve hedef gen bölgesi çoğaltılmıştır. İzole edilen genin dizi analizi yapılmış ve 4591 nükleotitten oluştuğu tespit edilmiştir. Referans diziye kıyasla 2 transisyonel, 14 transversiyonel ve 38 insersiyonel baza karşılık gelen 54 nükleotitten oluşan varyasyon bulunmuştur. Tüm nükleotit farklılıkları genin intron bölgelerinde tespit edilmiştir. Genin intron bölgelerinde yer alan putatif hormon yanıt elemanları (HRE) değerlendirildiğinde ise daha önce raporlanan dizi ile tamamen uyumlu olduğu görülmüştür. Çalışmada elde edilen veriler filogenetik ve gen aktarım çalışmalarında kullanılabilecek niteliktedir.

\section{Isolation and Characterization of Growth Hormone I Gene from Antalya Culture Population of Rainbow Trout (Oncorhynchus mykiss, Walbaum 1792)}

\begin{abstract}
In this study, the growth hormone I gene was isolated and characterized from the rainbow trout culture population reared in Antalya Province. For this purpose, 10 individuals from the culture population were sampled. Total DNA was isolated from the muscle tissue of fish. Primers used in the polymerase chain reaction were designed and the target gene region was amplified. Sequence analysis of the isolated gene was performed and it was determined that the gene consisted of 4591 nucleotides. Compared to the reference sequence, 54 nucleotide differences were found corresponding to 2 transitional, 14 transversional and 38 insertional bases. All nucleotide variations are located in the intron regions of the gene. When the putative hormone response elements (HRE) were evaluated, they were seen to be completely compatible with the previously reported sequence. The data obtained in this study can be used in phylogenetic and gene transfer studies.
\end{abstract}

Keywords: Growth hormone gene, rtghI, HRE, Rainbow trout, Oncorhynchus mykiss.

\footnotetext{
*Sorumlu Yazar: myilmaz@akdeniz.edu.tr
} 


\section{Giriş}

Genetik bilgi, genomda nükleik asit dizileri ile saklanır. Genler deoksiribonükleik asit (DNA) üzerinde genetik bilgiyi kodlayan birimlerdir ve genlerde ya bir protein kodlanmıştır ya da işlevsel RNA'nın bilgisi barındırılır (Gerstein et al., 2007; Hüttenhofer et al., 2005). Ökaryotlarda genler ekzon ve intron denilen alt birimlerden oluşmaktadır. Ekzonda eksprese edilecek proteine ait aminoasit dizisinin bilgisi 3'lü baz dizilerinden oluşan kodlar ile temsil edilmektedir (Nelson \& Cox, 2017). Ekzonlar oldukça korunmuş bölgelerdir. İntron bölgesinde yer alan baz dizileri ise ekzona göre daha fazla varyasyon gösterebilirler (Filiz \& Koç, 2011). Nükleik asit baz dizilerinde görülen bu varyasyonlar aynı türe ait popülasyonlar arasında ve türler arasındaki genetik farklılığın bir göstergesi olabilir ve filogenetik çalışmalarda yoğun olarak kullanılırlar (Klug et al., 2018; Kocher \& Carleton, 1997). Mitokondriyal genler (Xia, 2008) ve yapısal genler (Marins et al., 2003) bu amaçla kullanılabilen genlerdendir.

Genlerden aynı zamanda gen aktarım çalışmalarında da yararlanılmaktadır. Bu çalışmalarda aynı ya da farklı türlerden alınan DNA parçaları (düzenleyici dizi ve yapısal gen gibi) bir araya getirilerek rekombinant gen kaseti oluşturulmakta ve hedef türe aktarılmaktadır. Başarılı bir gen aktarımı sonucunda aktarılan genin kodladığı protein, hedef türde istenilen karaktere sahip soyların elde edilmesini sağlamaktadır (Yılmaz, 2012). Bu yolla hızlı büyüyen, hastalıklara dirençli, üretim koşullarına toleransl, istenilen renk ve et kalitesine sahip soylar elde edilebilmiştir.

Büyüme hormonu geni $(g h)$ balıklarda hem filogenetik çalışmalarda hem de gen aktarım çalışmalarında kullanılan yapisal bir gendir (Marins et al., 2003; Y1lmaz, 2012). Alabalıklarda büyüme hormonu $(\mathrm{GH})$; hipofiz bezinin anteriör kısmında somatotrof hücreleri tarafından üretilen ve yaklaşık 22 kDa büyüklüğünde tek zincirden oluşan bir polipeptittir (Mori et al., 2001). $\mathrm{Bu}$ hormon balığın büyüme, gelişim ve metabolizmasını düzenlemektedir (Rothan et al., 2014; Kaplan, 1999; Nicoll et al., 1999; Özcan-Gökçek \& Işık, 2020).

Salmonidlerde büyüme hormonunun moleküler yapısı ilk olarak chum salmon (O. keta) büyüme hormonunun izolasyonu, karakterizasyonu ve k1smi aminoasit dizisinin analiziyle aydınlatılmıştır (Sekine et al., 1985; Wagner et al., 1985). Ardından Agellon ve Chen (1986) gökkuşağı alabalığı GH aminoasit dizisini ortaya koymuşlardır. Bunu takiben gökkuşağı alabalığından $g h \mathrm{I}$ geni izole edilerek tanımlanmıştır (Agellon et al., 1988; Björnsson 1997).

Glukokortikoid, tiroid ve retinoik asit gibi hormonlar, nükleer reseptörler aracılığıyla fizyolojik yanıtlar ortaya çıkarmaktadırlar. Büyüme hormonu geninin ekspresyonunun düzenlenmesinde rol alan sinyal reseptör kompleksi, DNA sekansının belirli bir bölgesine (hormone response element, HRE) bağlanmak ve seçilen genlerin ekspresyonunu düzenlemek üzere bir transkripsiyon faktörü olarak görev alır (Barlow et al., 1986; Slater et al., 1985).

Yapılan bu çalışmada, Antalya ilinde bulunan bir çiftlikte üretilen gökkuşağı alabalığı popülasyonundan ghI geni izole ve karakterize edilerek literatürde rapor edilen (Yang et al., 1997) referans dizi ile benzerlik/farklılıkları araştırılmıştır. Tespit edilen varyasyonların putatif HRE bölgelerinde değişikliğe neden olup olmadığı değerlendirilmiştir. Böylece filogenetik ve e-ISSN: 2148-2683 gen aktarım çalışmalarında kullanılabilecek kıymetli bir veri seti oluşturulmuştur.

\section{Materyal ve Metot}

\subsection{Balıkların Temini}

Gökkuşağı alabalığı örnekleri (10 adet) Antalya İli’nde yetiştiricilik faaliyeti sürdüren bir çiftlikten temin edilmiştir. Örneklenen bireyler ayrı ayrı PE torbalara yerleştirilip etiketlenmiştir. Buzla soğutulmuş kutuda Akdeniz Üniversitesi $\mathrm{Su}$ Ürünleri Fakültesi Yetiştiricilik Laboratuvarına taşınmıştır. Laboratuvarda dorsal yüzgeçlerinin hemen gerisinden laterale yakın bölgeden alınan kas doku parçaları moleküler işlemler uygulanıncaya kadar $-40^{\circ} \mathrm{C}$ derin dondurucuda steril tüpler içinde muhafaza edilmiştir.

\subsection{DNA İzolasyonu}

Toplam genomik DNA izolasyonu için yaklaşık $5 \mathrm{mg}$ doku örneği ve CTAB DNA izolasyon protokolü (Freeland, 2008) kullanılmıştır. Kalıp DNA miktarları ve kalitesi nanodrop spektrofotometre (Thermo) ile belirlenmiştir (Kennedy \& Oswald, 2011). İzole edilen kalıp DNA kullanılana kadar $-20{ }^{\circ} \mathrm{C}$ derin dondurucuda saklanmıştır.

\subsection{Primer Dizaynı}

Yang et al. (1997) tarafindan bildirilen gökkuşağı alabalığ1 GHI geni (rtghI) nükleotit dizisi izolasyon çalışmalarında referans dizi olarak kullanılmıştır. $r$ tghI genine ait dizisinin 5' ve 3' ucunda yer alan bölgelerin Temel Bölgesel Hizalama Arama Aracı (Basic Local Alignment Search Tool, BLAST) yardımı ile GenBank veritabanında bulunan diziler ile uyumlarına bakılmıştır. Yapılan taramada yakın akraba türlere ait dizilerin korunmuş bölgeleri primer dizaynı için kullanılmıştır.

rtghI geni DNA bölgesinin PCR yöntemi ile çoğaltılması için gerekli olan primerler hedef DNA bölgelerinin komplementer dizilerine karşıllı gelen $\mathrm{F}$ 5, GCACTTTCAAGCTAAGTAACCATCCTTGGC 3' ve R 5' GCAGCAGACCCATGTTTGGAAAGTCTG 3' dizilerden oluşturulmuştur. PCR reaksiyonunda kullanılacak primerlerin primer uzunluğu, GC yüzdesi, bağlanma ve çözünme sıcaklıkları, 5' uç kararlılığı, 3' uç spesifikliği gibi kriterleri karşılayacak şekilde (Dieffenbach et al., 1995) dizayn edilmiştir. Primerler hizmet alımı yolu ile sentezletilmiştir (GenScript).

\subsection{Polimeraz Zincir Reaksiyonu Koşulları}

Hedeflenen $r \operatorname{tg} h \mathrm{I}$ DNA bölgelerinin polimeraz zincir reaksiyonu (PZR) ile çoğaltımı için DNA polimeraz (ExTaq, Takara Inc.) üretici firmanın önerileri doğrultusunda kullanılmıştır. Reaksiyon farklı denatürasyon, primer bağlanma ve sentez sıcaklıkları denenerek optimize edilmiştir (Kennedy \& Oswald, 2011). PCR reaksiyonu için kullanılan termal döngü koşulları başlangıç denatürasyonu $95^{\circ} \mathrm{C}$ 'de 3 dak, denatürasyon $98^{\circ} \mathrm{C}$ 'de $10 \mathrm{sn}$, primerlerin bağlanmas $55^{\circ} \mathrm{C}$ 'de $10 \mathrm{sn}$., DNA sentezi $72^{\circ} \mathrm{C}$ 'de 5,5 dak ve son uzatma $72^{\circ} \mathrm{C}$ 'de 5 dak olarak uygulanmıştır. Denatürasyon, bağlanma ve sentez basamakları 30 döngü olacak şekilde reaksiyon sürdürülmüştür.

\subsection{PZR Ürünlerinin Kalitesinin Belirlenmesi, Saflaştırılması ve Dizi Analizi}

Total DNA izolatlarının kalitesini belirlemek için örnekler $\% 0,8$ 'lik, PZR ürünleri görmek için örnekler $\% 2$ 'lik agaroz jelde 
yürütülerek görüntülenmiştir. Her iki jelin elektroforezi için 8 volt $/ \mathrm{cm}^{2}$ 'lik bir elektrik alan kullanılmıştır (Brody \& Kern, 2004). Örneklerin moleküler büyüklüklerini tespit etmek için jellerin bir kuyucuğuna $1 \mathrm{~kb}$ Ladder (Bioron) yüklenmiştir.

Hedef diziye ait PZR ürünü jel ekstraksiyon yöntemi ile saflaştırılmıştır. $\mathrm{Bu}$ amaçla ticari bir kit (Nanohelix), üretici firmanın önerdiği protokol doğrultusunda kullanılmıştır. İzole edilen genin dizi analizi hizmet alımı yoluyla (Genscript) yaptırılmıştır. Dizi analizinde 1,3-7 kb uzunluğundaki diziler için uygun olan "primer yürütme" tekniği kullanılmıştır (Bhatia \& Dahiya, 2015; Clark \& Pazdernik, 2013).

\subsection{Kullanılan Yazılımlar}

$\mathrm{Bu}$ çalışmalar sırasında referans dizinin baz uyumları ile dizayn edilen primer dizilerinin uygunluğunun kontrolü için BLAST, BioEdit (Hall, 1999), CLUSTAL W (Thompson et al., 1994) ve FastPCR Professional (Kalendar et al., 2009) yazılımlarından yararlanılmıştır.

\section{Araştırma Sonuçları ve Tartışma}

Büyüme hormonu I genine ait özgün nükleotit dizisi türler arasında farklılık gösterebilmektedir. Gökkuşağı balıklarında yaklaşık 4500 baz çiftine sahip olan bu gen (Şekil 1) Labeo türlerinde yaklaşık 2500 baz çifti büyüklüktedir (Rajesh \& Majumdar, 2007).

Daha önceki çalışmalarda (Yang et al., 1997; Agellon et al., 1988) rapor edildiği gibi rtghI geni 6 ekzon ve 5 introndan oluşmaktadır. $\mathrm{Bu}$ yapı baramundi (Yowe \& Epping, 1995), atlantik salmon (Johansen et al., 1989; Male et al., 1992), tilapia (Ber \& Daniel, 1993) ile benzerlik gösterirken, 5 ekzon ve 4 introna sahip olan Labeo türlerinden (Rajesh \& Majumdar, 2007) farklıdır. Genin kodladığı amino asit sayısı da türler arasında farklılıklar göstermektedir. Çalışmamızda izole edilen genin referans dizi ile yapılan kıyaslamasında, daha önce rapor edildiği gibi, 6 ekzonda 630 baz çiftinden oluşan açık okuma çerçevesinde 210 amino asit kodladığı görülmüştür. Ancak, büyüme hormonu geni Labeo roita 'da 4. ekzonda görülen 9 baz çiftlik delesyonla 207 amino asit kodlamaktadır (Rajesh \& Majumdar, 2007).

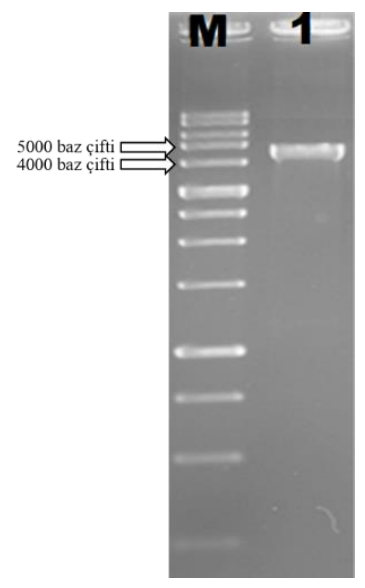

Şekil 1. rtghI geni PCR ürününün agaroz jel görüntüsü (M: Markır)

Çalışmamızda izole edilen Antalya kültür popülasyonuna ait rtghI geninin dizi analizi sonuçları genin 4591 baz çiftine sahip olduğunu göstermektedir. Yang et al. (1997) ise rtghI geninin 4552 baz çiftlik bir nükleotit dizisinden oluştuğunu bildirmişlerdir. Genin başlangıç (ATG) ve dur (TAG) kodları arasında yer alan nükleotit dizisi değerlendirildiğinde, bu iki dizinin \%98,78 oranda birbiriyle uyumlu olduğu belirlenmiştir. Tespit edilen \%1,22 oranındaki uyumsuzluk genin intron bölgelerinde yer almaktadır ve 2 transisyonel, 14 transversiyonel ve 38 insersiyonel baza karşılık gelen 54 nükleotit varyasyonu içermektedir. Genin sadece ekzon bölgeleri karşılaştırıldığında, bu iki dizinin \%100 uyumlu olduğu görülmüştür (Tablo 1).

Ayrıca Antalya ilinde üretilen kültür popülasyonundan izole edilen rtghI geninde yer alan tüm intronların başlangıç ve bitiş dinükleotidleri klasik korunmuş GT/AG uçbirleşme bölgeleri (Mount, 1982) ile uyum içerisindedir.

Tablo 1. Antalya ilinde üretilen kültür popülasyonundan izole edilen rtghI geni nükleotid dizisinin referans dizi (Yang et al., 1997) ile karşılaştırması

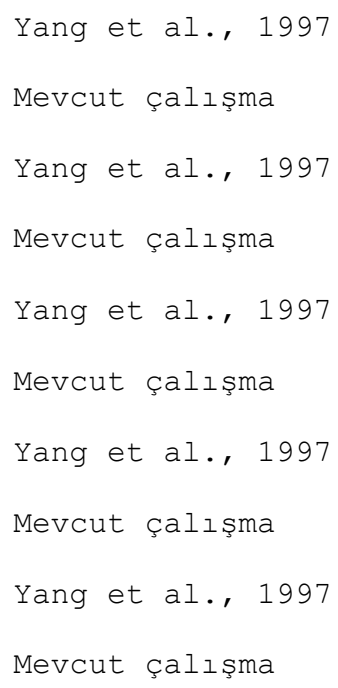

1 ACATACTCAACCGACCACCGCACTTTCAAGCTAAGTAACCATCCTTGGCA || |||||||||||||||||||||||||||||||||||||||||||||||||||||| 1 ACATACTCAACCGACCACCGCACTTTCAAGCTAAGTAACCATCCTTGGCA

51 ATTAAGAGTAAAAATGGGACAAGgtaagcctgcttttctgtctatttat ||||||||||||||||||||||||||||||||||||||||||||||||||||||| 51 ATTAAGAGTAAAAATGGGACAAGgtaagcctgcttttctgtctatttat $\overline{\mathrm{BSS}}$

101 tttttcagtgggaagtcagtgraccatttagtacaatttaactactgcta || |||||||||||||||||||.|||||||||||||||||||||||||||| 101 tttttcagtgggaagtcagtgtaccatttagtacaatttaactactgcta

151 tgaggttataatctattgacacagaaccacctgctttaacaacctaacta ||||||||||||||||||||||||||||||||||||||||||||||||||| 151 tgaggttataatctattgacacagaaccacctgctttaacaacctaacta

201 tgtgatccataacatttacatttgagtcatttagcagacactcttatcca |||||||||||||||||||||||||||||||||||||||||||||||||||||| 201 tgtgatccataacatttacatttgagtcatttagcagacactcttatcca 
Yang et al., 1997

Mevcut çalışma

Yang et al., 1997

Mevcut çalışma

Yang et al., 1997

Mevcut çalışma

Yang et al., 1997

Mevcut çalışma

Yang et al., 1997

Mevcut çalışma

Yang et al., 1997

Mevcut çalışma

Yang et al., 1997

Mevcut çalışma

Yang et al., 1997

Mevcut çalışma

Yang et al., 1997

Mevcut çalışma

Yang et al., 1997

Mevcut çalışma

Yang et al., 1997

Mevcut çalışma

Yang et al., 1997

Mevcut çalışma

Yang et al., 1997

Mevcut çalıșa

Yang et al., 1997

Mevcut çalışma

Yang et al., 1997

Mevcut çalışma

Yang et al., 1997

Mevcut çalışma

Yang et al., 1997

Mevcut çalışma

Yang et al., 1997

Mevcut çalışma
251 ga-cgacttacatgagcaattggggttacgtgccttgctcaagggcacat || |||||||||||||||||||||||||||||||||||||||||||||||| 251 gagcgacttacatgagcaattggggttacgtgccttgctcaagggcacat

300 cagatttctcacctagtcagctctggggttgaaaccagtaacgacccagc || || || || || || || ||||||||||||||||||||||||||||||||||| 301 cagatttctcacctagtcagctctggggttgaaaccagtaacgacccagc

350 gctcttaacccgcta-gctattggtgtacgatggctgagaaaatcttacc |||||||||||||||||||||||||||||||||||||||||||||||| $\mid$

351 gctcttaacccgctaggctattggtgtacgatggctgagaaaatcttacc

399 aatgtatctcaccataattcgacttactcgttttctacatttcttatttg ||||||||||||||||||||||||||||||||||||||||||||||||||

401 aatgtatctcaccataattcgacttactcgttttctacatt tcttatttg

449 aatctctctttagTGTTTCTGCTGATGCCAGTCTTACTGGTCAGTTGTT |||||||||||||||||||||||||||||||||||||||||||||||||| 451 aatctctctttagTGTTTCTGCTGATGCCAGTCTTACTGGTCAGTTGTT

499 TCCTGAGTCAAGGGGCAGCGATAGAAAACCAACGGCTCTTCAACATCGCG

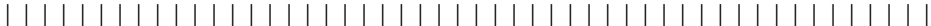

501 TCCTGAGTCAAGGGGCAGCGATAGAAAACCAACGGCTCTTCAACATCGCG

549 GTCAGCCGGGTGCAACATCTCCACCTATTGGCTCAGAAAATGTTCAATGA

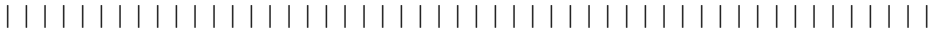

551 GTCAGCCGGGTGCAACATCTCCACCTATTGGCTCAGAAAATGTTCAATGA GRE, $\mathrm{PRE}, \overline{\mathrm{ARE}}, \overline{\mathrm{MRE}}$

599 CTTTgtaagacagctttgaatcttcttggacatatcaaatagtgtatc ||||||||||||||||||||||||||||||||||||||||||||||||||

601 СтTTgtaagacagcttttgaatcttctttggacatatcaaatagtgtatc

649 aatgattgttcttcttcttgtagacagtgtcctcagcacacaaccctcgt ||||||||||||||||||||||||||||||||||||||||||||||||||

651 aatgattgttcttcttcttgtagacagtgtcctcagcacacaaccctcgt GRE, PRE, ARE, MRE

699 ggctaaaaaatctctctctcccttgtgattttgtgcagGACGGTACCC || || |||||||||||||||||||||||||||||||||||||||||||||||||||

701 ggctaaaaaatctctctctcccttgtgattttgtgcagGACGGTACCC

749 TGTTGCCTGATGAACGCAGACAGCTGAACAAGATATTCCTGCTGGACTTC | | | | | | | | | | | | | | | | | | | | | | | | | | | | | | | | | | | | | | | | | | | | | | | | | |

751 TGTTGCCTGATGAACGCAGACAGCTGAACAAGATATTCCTGCTGGACTTC

799 TGTAACTCTGACTCCATCGTGAGCCCAGTCGACAAGCACGAGACTCAGAA

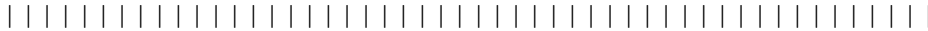
801 TGTAACTCTGACTCCATCGTGAGCCCAGTCGACAAGCACGAGACTCAGAA

849 GAGTTCAgtaagtaacctggccgaaacacttacgcatgttatgccettta ||||||||||||||||||||||||||||||||||||||||||||||||||

851 GAGTTCAgtaagtaacctggccgaaacacttacgcatgttatgccettta

899 gaaccatataaagtgtcaaatcgctattcaccttaatatgaactcctcc |||||||||||||||||||||||||||||||||||||||||||||||||| 901 gaaccatataaagtgtcaaatcgctattcaccttaaatatgaactcctcc

949 atgatgcaagattccaaaataaataata--taataataatttgaacaat ||||||||||||||||||||||||||||$|\ldots||\ldots||||||||||| \mid$ 951 atgatgcaagattccaaaataaataatagggcatctcaatttgaacaat

997 cgatagaacttacggtagtcattagttattgggcaagcagaccaccaatt |||||||||||||||||||||||||||||||||||||||||||||||||| 1001 cgatagaacttacggtagtcattagttattgggcaagcagaccaccaatt $\mathrm{ERE}$

1047 atgtaaactccaatttctaattttacattttaatttgatttgaacctta |||||||||||||||||||||||||||||||||||||||||||||||||| 1051 atgtaaactccaatttctaattttacattttaatttgatttgaacctta

1097 tttaactaggcaagtcagttaagaacaaattctcatttataatgacaagc ||||||||||||||||||||||||||||||||||||||||||||||||||

1101 tttaactaggcaagtcagttaagaacaaattctcatttataatgacaagc 
Yang et al., 1997

Mevcut çalışma

Yang et al., 1997

Mevcut çalışma

Yang et al., 1997

Mevcut çalışma

Yang et al., 1997

Mevcut çalışma

Yang et al., 1997

Mevcut çalışma

Yang et al., 1997

Mevcut çalışma

Yang et al., 1997

Mevcut çalışma

Yang et al., 1997

Mevcut çalışma

Yang et al., 1997

Mevcut çalışma

Yang et al., 1997

Mevcut çalıșma

Yang et al., 1997

Mevcut çalışma

Yang et al., 1997

Mevcut çalışma

Yang et al., 1997

Mevcut çalışma

Yang et al., 1997

Mevcut çalışma

Yang et al., 1997

Mevcut çalışma

Yang et al., 1997

Mevcut çalışma

Yang et al., 1997

Mevcut çalışma
1147 agaggcagaatcatgcatggctattgagtgatgcagcagtctaaggcact |||||||| . ||||||||||||.||||||||||||||||||||||||||||

1151 agaggcagcctcatgcatggctcttgagtgatgcagcagtctaaggcact

1197 acatctcagtg-cagaggtgtcactgcagaccctggtatgattccagact |||||||||||||||||||||||||||||||||||||||||||||||| $\mid$

1201 acatctcagtgccagaggtgtcactgcagaccctggtatgattccagact

1246 gtatttcaaacggctgtgattgtgagt-ccatagggcggcacacaattct ||||||||||||||||||||||||||||| ||||||||||||||||||||||||

1251 gtatttcaaacggctgtgattgtgagtcccatagggcggcacacaattct

1295 cccagcgtcgttagggtttggccggggttgcaatacctcagtgtcttcaa ||||||||||||||||||||||||||||||||||||||||||||||||||||||||

1301 cccagcgtcgttagggtttggccggggttgcaatacctcagtgtcttcaa

1345 ctaaaggtagataaacaaccacatttcacaatcattgcaagtaaacca || ||||||||||||||||||||||||||||||||||||||||||||||||||

1351 ctaaaggtagataaacaaccacatttcacaatcattgcaagtaaacca

1395 tcactgtctaatcggtggtttctctacgtctacattctccgttttgtgct |||||||||||||||||||||||||||||||||||||||||||||||||||||

1401 tcactgtctaatcggtggtttctctacgtctacattctccgttttgtgct

1445 tttctgtacaggaaacccgccccaaagtatttcactcaatcatgtaaa |||||||||||||||||||||||||||||||||||||||||||||||||||||

1451 tttctgtacaggaaacc gccccaaagtattttcactcaatcatgtaaa

1495 tagggcatctcaagctgtacaatacaactcaacttcattttccaataatc ||||||||||||||||||||||||||||||||||||||||||||||||||||||||

1501 tagggcatctcaagctgtacaatacaactcaacttcattttccaataatc

1545 tgtggttetctacatcttcacacacagGTCCTGAAGCTGCTCCATATCT |||||||||||||||||||||||||||||||||||||||||||||||||||||||| 1551 tgtggttetctacatcttcacacacagGTCCTGAAGCTGCTCCATATCT

1595 CTTTCCGTCTGATTGAATCCTGGGAGTACCCTAGCCAGACCCTGATCATC |||||||||||||||||||||||||||||||||||||||||||||||||||||||

1601 CTTTCCGTCTGATTGAATCCTGGGAGTACCCTAGCCAGACCCTGATCATC

1645 TCCAACAGCCTAATGGTCAGAAATGCCAACCAGATCTCTGAGAAGCTCAG ||||||||||||||||||||||||||||||||||||||||||||||||||||||||

1651 TCCAACAGCCTAATGGTCAGAAATGCCAACCAGATCTCTGAGAAGCTCAG

1695 CGACCTCAAAGTGGGCATCAACCTGCTCATCACGgtaaagaaaggaggga ||||||||||||||||||||||||||||||||||||||||||||||||||||||||

1701 CGACCTCAAAGTGGGCATCAACCTGCTCATCACGgtaagaaggaggga

1745 gaacaatgaccatttgtggtgtcacactttgtgcactgtaaactccaagg ||||||||||||||||||||||||||||||||||||||||||||||||||||||||

1751 gaacaatgaccatttgtggtgtcacactttgtgcactgtaaactccaagg GRE, PRE, ARE, MRE

1795 catttttaactcaaatacttctagtaagttgaactcaaagtcaatgaaaa |||||||||||||||||||||||||||||||||||||||||||||||||||||

1801 cattttaactcaatacttctagtaagttgaactcaagtcaatgaaaa

1845 atccttattgcttaaatgtttatgtggtactggctcaaaaccaaatgag || |||||||||||||||||||||||||||||||||||||||||||||||||||

1851 atccttattgcttaaatgtttatgtggtactggctcaaaaccaaatgag

1895 aagtcacatcaatgcaattttttaaagttataacaaattcacttttacca ||||||||||||||||||||||||||||||||||||||||||||||||||||||

1901 aagtcacatcaatgcaattttttaagttataacaattcacttttacca

1945 agcatgctctactgcaggtaga-tttttttaaatgtacagaacattgagt |||||||||.|||||||||||| ||||||||||||||||||||||||||||| 1951 agcatgctcaactgcaggtagattttttttaatgtacagaacattgagt 


\section{Avrupa Bilim ve Teknoloji Dergisi}

Yang et al., 1997

Mevcut çalışma

Yang et al., 1997

Mevcut çalışma

Yang et al., 1997

Mevcut çalışma

Yang et al., 1997

Mevcut çalışma

Yang et al., 1997

Mevcut çalışma

Yang et al., 1997

Mevcut çalışma

Yang et al., 1997

Mevcut çalışma

Yang et al., 1997

Mevcut çalışma

Yang et al., 1997

Mevcut çalışma

Yang et al., 1997

Mevcut çalışma

Yang et al., 1997

Mevcut çalışma

Yang et al., 1997

Mevcut çalışma

Yang et al., 1997

Mevcut çalışma

Yang et al., 1997

Mevcut çalışma

Yang et al., 1997

Mevcut çalışma

Yang et al., 1997

Mevcut çalışma

Yang et al., 1997

Mevcut çalışma

Yang et al., 1997

Mevcut çalışma
1994 gattgattcatttatgctacacaagatatataacatacatgtttcaac || ||||||||||||||||||||||||||||||||||||||||||||||||| 2001 gattgattcattttatgctacacaaagatatataacatacatgtttcaac

2044 attttcacaagatgaacaagttaccagaattttgcaaactcaacttg-a || || || || || || || ||||||||||||||||||||||||||||||||| | 2051 attttcacaagatgaacaagttaccagaattttgcaaactcaacttgca

2093 cgcctgatgtggcctgtataccatgagttgcaggccactgtattagggta

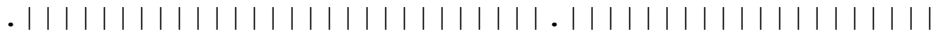

2101 ggcetgatgtggcctgtataccatgagtttcaggccactgtattagggta

2143 aagctacacctcaaatatggtattatgagataagtcatgtattgttgta |||||||||||||||||||||||||||||||||||||||||||||||||| 2151 aagctacacctcaaatatggtattatgagataagtcatgtattgttgta

2193 aagacttgaatt---------------------------acttgaaggcc ||||||||||||$\quad|||||||||| \mid$

2201 aagacttgaattctaatgataatatttgcctaggaattcacttgaaggcc

2216 acaggactgaaatgaatgacaacagccatgtctctgtcactaacatata

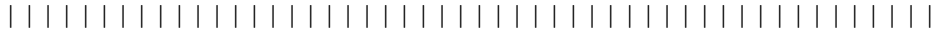
2251 acaggactgaaatgaatgacaacagccatgtctctgtcactaacatata

2266 cagtcatgggtgataactacacttcactcaaaaaggccaggcacactggg || |||||||||||||||||||||||||||||||||||||||||||||||| 2301 cagtcatgggtgataactacacttcactcaaaaggccaggcacactggg

2316 aaattatatttgagacgtggcttagtgggggcattactaaaaatgtcaa ||||||||||||||||||||||||||||||||||||||||||||||||||

2351 aaattatatttgagacgtggcttagtgggggcattactaaaaatgtcaa

2366 gctgatacaactcaaatctggacacatcacagggtgactctataggtttg |||||||||||||||||||||||||||||||||||||||||||||||||| 2401 gctgatacaactcaaatctggacacatcacagggtgactctataggtttg

2416 agtaatgactgactataacatcactttaagtaactgcagtcagattctgt || || ||||||||||||||||||||||||||||||||||||||||||||||||||| 2451 agtaatgactgactataacatcactttaagtaactgcagtcagattctgt

2466 atattaagtgcaacaggtttcctaaaagtgttgagtaatggcagcacat

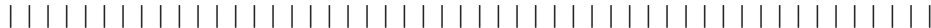
2501 atattaagtgcaacaggtttcctaaaagtgttgagtaatggcagcacat

2516 tggggtttacagtgacatgaaagggaaatacctttatgctttcctagtta || || |||||||||||||||||||||||||||||||||||||||||||||| 2551 tggggtttacagtgacatgaaagggaaatacctttatgctttcctagtta

2566 gaaagcatagtgtaggaccacgtatgcctcttctcagcagatctttcagg ||||||||||||||||||||||||||||||||||||||||||||||||||

2601 gaaagcatagtgtaggaccacgtatgcctcttctcagcagatctttcagg

2616 gcttacattgtgatgtggtaactgaccttatccatcatcgtgattatat |||||||||||||||||||||||||||||||||||||||||||||||||| 2651 gctttacattgtgatgtggtaactgaccttatccatcatcgtgattatat

2666 cagtgacaccccattcaatgactgaatatcgccccattcaaggacattta || || |||||||||||||||||||||||||||||||||||||||||||||||||

2701 cagtgacaccccattcalgactgaatatcgccccattcaaggacattta

2716 tgcatgtgtctttgctacgtgtgctttcagaaaggcccaataaacaaat

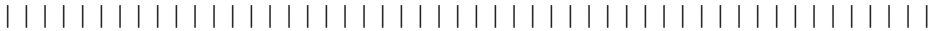
2751 tgcatgtgtctttgctacgtgtgcttcagaaaggcccaataaacaat

2766 attgatatgcacacatccaccccaccatgcatctctctctgtctcccaca || || || || |||||||||||||||||||||||||||||||||||||||||| 2801 attgatatgcacacatccaccccaccatgcatctctctctgtctcccaca

2816 gGGGAGCCAGGATGGCGTACTGAGCCTGGATGACAATGACTCTCAGCAGC ||||||||||||||||||||||||||||||||||||||||||||||||||

2851 gGGGAGCCAGGATGGCGTACTGAGCCTGGATGACAATGACTCTCAGCAGC
2043

2050

2092

2100

2142

2150

2192

2200

2215

2250

2265

2300

2315

2350

2365

2400

2415

2450

2465

2500

2515

2550

2565

2600

2615

2650

2665

2700

2715

2750

2765

2800

2815

2850

2865

2900 
Yang et al., 1997

Mevcut çalışma

Yang et al., 1997

Mevcut çalışma

Yang et al., 1997

Mevcut çalışma

Yang et al., 1997

Mevcut çalışma

Yang et al., 1997

Mevcut çalışma

Yang et al., 1997

Mevcut çalışma

Yang et al., 1997

Mevcut çalışma

Yang et al., 1997

Mevcut çalışma

Yang et al., 1997

Mevcut çalışma

Yang et al., 1997

Mevcut çalışma

Yang et al., 1997

Mevcut çalışma

Yang et al., 1997

Mevcut çalışma

Yang et al., 1997

Mevcut çalışma

Yang et al., 1997

Mevcut çalışma

Yang et al., 1997

Mevcut çalışma

Yang et al., 1997

Mevcut çalışma

Yang et al., 1997

Mevcut çalışma
2866 TGCCCCCCTACGGGAACTACTACCAGAACCTGGGGGGCGACGGAAACGTC ||||||||||||||||||||||||||||||||||||||||||||||||||||||||

2901 TGCCCCССTACGGGAACTACTACCAGAACCTGGGGGGCGACGGAAACGTC

2916 AgGAGGAACTACGAGTTGTTGGCTTGCTTCAAGAAGGACATGCACAAGgt

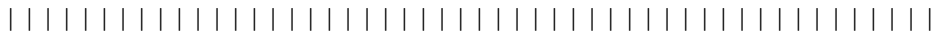
2951 AGGAGGAACTACGAGTTGTTGGCTTGCTTCAAGAAGGACATGCACAAGgt RXR-RAR

2966 cggcaaccatgttgccttcaatatcatgtgccttcctgtattttctacag .. ||||||||||||||||||||||||||||||||||||||||||||||||||||||||||

3001 gcgcaaccatgttgccttcaatatcatgtgccttcctgtattttctacag

3016 tgcgttgttttttgtgttctctattgcaaagtattgttagtaaataact ||||||||||||||||||||||||||||||||||||||||||||||||||||||| 3051 tgcgttgttttttgtgttctctattgcaaagtattgttagtaaataact

3066 cacggacactagagaagc-ttaaccaagtttaattcttcccaaaggttct |||||||||||||||||| ||||||||||||||||||||||||||||||||

3101 cacggacactagagaagctttaaccaagtttaattcttcccaaaggttct

3115 gtaccgctgtaatcagacagcaaacatttctcaatccacagtcatatac |||||||||||||||||||||||||||||||||||||||||||||||||||||||||

3151 gtaccgctgtaatcagacagcaaacatttctcaatccacagtcatatac

3165 atcttacttaaaacactcctccttcttcaatccttacagtttatggctcc |||||||||||||||||||||||||||||||||||||||||||||||||||||||

3201 atcttacttaaacactcctccttcttcaatccttacagtttatggctcc

3215 acaggaagctaataaagaggataacaggacaacaaacatttattgctgcc ||||||||||||||||||||||||||||||||||||||||||||||||||||||||||| 3251 acaggaagctaataaagaggataacaggacaacaacatttattgctgc

3265 ttcagagaatctgtcctcacctcctgacctcaacccetcatctaatccac || |||||||||||||||||||||||||||||||||||||||||||||||||||||

3301 ttcagagaatctgtcctcacctcctgacctcaacccctcatctaatccac

3315 agatgtccattgttttttttcggagaaccattaagttctgacatgaccca ||||||||||||||||||||||||||||||||||||||||||||||||||||||

3351 agatgtccattgtttttttcggagaaccattaagttctgacatgaccca

3365 gtttctttcatttactatctcaatgatcaacgtttagccgattccaacag |||||||||||||||||||||||||||||||||||||||||||||||||||||||||

3401 gtttcttcatttactatctcaatgatcaacgtttagccgattccaacag

3415 tatctttgggtcttaacctatatattattactattattgttcattgatc ||||||||||||||||||||||||||||||||||||||||||||||||||||||

3451 tatctttgggtcttaacctatatattattactattattgttcattgatc

3465 aagactgttctcgag-aaggtctggtgacctagaacacacacattaaaat |||||||||||||||| |||||||||||||||||||||||||||||||||||||||

3501 aagactgttctcgagaaggtctggtgacctagaacacacacattaaat

3514 gtgtcaactataacccattcttcttttttgaaccCCcgagGTCGAGACC ||||||||||||||||||||||||||||||||| ||||||||||||||||||||||| 3551 gtgtcaactataacccattcttcttttttcaaccCccgagGTCGAGACC RXR-RAR, RXR-TR, RXR-RXR，TR-TR，TR-RAR

3564 TACCTGACCGTCGCCAAGTGCAGGAAGTCACTGGAGGCCAACTGCACTCT |||||||||||||||||||||||||||||||||||||||||||||||||||||||||

3601 TACCTGACCGTCGCCAAGTGCAGGAAGTCACTGGAGGCCAACTGCACTCT

$$
\text { TR-RAR, RXR-TR }
$$

3614 GTAGACGTGGGCTGGAGAGGCAGCCAGCAAGAGCCTGTCTCCAGGGTTCG |||||||||||||||||||||||||||||||||||||||||||||||||||||||

3651 GTAGACGTGGGCTGGAGAGGCAGCCAGCAAGAGCCTGTCTCCAGGGTTCG $\overline{\mathrm{DUR}}$

3664 GTTTCCCAGATACAGATTAGGCCTTGCCCTGCACTGAGGTGCATTTTCAA ||||||||||||||||||||||||||||||||||||||||||||||||||||| 3701 GTTTCCCAGATACAGATTAGGCCTTGCCCTGCACTGAGGTGCATTTTCAA 


\section{Avrupa Bilim ve Teknoloji Dergisi}

Yang et al., 1997

Mevcut çalışma

Yang et al., 1997

Mevcut çalışma

Yang et al., 1997

Mevcut çalışma

Yang et al., 1997

Mevcut çalışma

Yang et al., 1997

Mevcut çalışma

Yang et al., 1997

Mevcut çalışma

Yang et al., 1997

Mevcut çalışma

Yang et al., 1997

Mevcut çalışma

Yang et al., 1997

Mevcut çalışma

Yang et al., 1997

Mevcut çalışma

Yang et al., 1997

Mevcut çalışma

Yang et al., 1997

Mevcut çalışma

Yang et al., 1997

Mevcut çalışma

Yang et al., 1997

Mevcut çalışma

Yang et al., 1997

Mevcut çalışma

Yang et al., 1997

Mevcut çalışma

Yang et al., 1997

Mevcut çalışma

Not: Ekzonlar büyük harfle, intronlar küçük harfle, kodlama bölgesi koyu harflerle belirtilmiştir. Putatif HRE’lerin altı kesikli çizgi, başlama ve dur kodlarının altları çift çizgi ve putatif Poly A sinyal dizisi altı çizili olarak verilmiştir. BŞL: Başlama kodu, DUR: Dur kodu, Poly A: Poliadenilasyon sinyali, GRE: glukokortikoid yanıt elementi, PRE: progesteron yanıt elementi, ARE: androjen yanıt elementi, MRE: mineralokortikoid hormon yanıt elementi, ERE: Östrojen yanıt elementi, RXR: retinoid $\mathrm{X}$ reseptörü, RAR: retinik asit reseptörü, TR: tiroid hormon reseptörü.
3714 TTGAGATTCTCCATTAAACATGCTTTTCAGTCTAGAGTAATTTTATTTTG

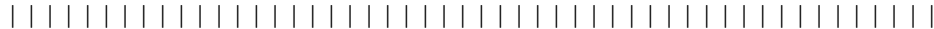
3751 TTGAGATTCTCCATTAAACATGCTTTTCAGTCTAGAGTAATTTTATTTTG

3764 GATCTAGTAGAGCCTGACTCCAGGGGTTTTCAGGCATTTGCCTTTTTTTC

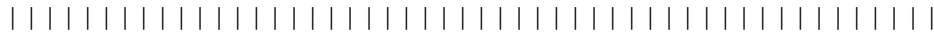
3801 GATCTAGTAGAGCCTGACTCCAGGGGTTTTCAGGCATTTGCCTTTTTTTC

3814 TCTGAAATCAACAACAACACTTTCTATATTGACTCTATCACTCTGAGCTA

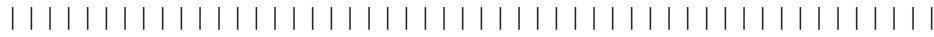
3851 TCTGAAATCAACAACAACACTTTCTATATTGACTCTATCACTCTGAGCTA

3864 CCATTGATTAGTACATTTACAGAAAAGGTTATTAAATGTTTATTTAGAT ||||||||||||||||||||||||||||||||||||||||||||||||||

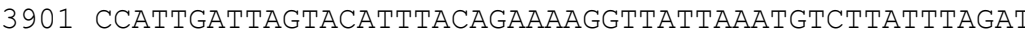

3914 ATATGGTTCATGGCGGTGCTACTGTTTATGCATACGTTAATATTTAGGGG |||||||||||||||||||||||||||||||||||||||||||||||||| 3951 ATATGGTTCATGGCGGTGCTACTGTTTATGCATACGTTAATATTTAGGGG

3964 TGAAATGAGAACTTGTAGAGCTCCAAGCTTTTGGATAATATATTTTAGAG |||||||||||||||||||||||||||||||||||||||||||||||||||| $\mid$ 4001 TGAAATGAGAACTTGTAGAGCTCCAAGCTTTTGGATAATATATTTTAGAG

4014 TAATTCCTTTACGTATTTTCATTCCTTAATCTTATTGTTTGAAACTAAT ||||||||||||||||||||||||||||||||||||||||||||||||||||||

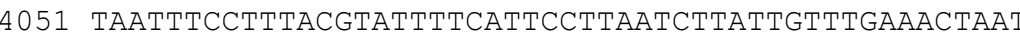

4064 AGTGATTCATGTTTCAATAAAGATGTTCTTCTCTGCAgcacataatctct |||||||||||||||||||||||||||||||||||||||||| $\mid$ ||||||| 4101 AGTGATTCATGTTTCAATAAAGATGTTCTTCTCTGCAgcacatgatctct Poly A

4114 tggcttctatttaatatcttcaaatcaacatttttacaagttcctagc |||||||||||||||||||||||||||||||||||||||||||||||||| 4151 tggcttctatttaatatcttccaatcaacattttttacaagttcctagc

4164 cccaacattcctatggtgtctctcggacaacttagggctggattcatcc || || ||||||||||||||||||||||||||||||||||||||||||||||||||| 4201 cccaacattcctatggtgtctctcggacaacttagggctggattcaatcc

4214 gtatcgcagatgctccatttgaaatgtaaaggcaatgttcctgcgttcgc

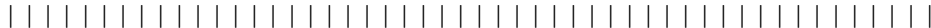
4251 gtatcgcagatgctccatttgaaatgtaaaggcaatgttcctgcgttcgc

4264 -gagactgcattcacttcaaacgctgcatatgtcggctcaatcggaatt

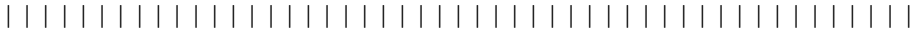
4301 ggagactgcattcacttcaaacgctgcatatgtcggctcaatcggaatt

4313 acctgaaaatgttatacggttcttcagcgatacggattgaatccagccc |||||||||||||||||||||||||||||||||||||||||||||||||| 4351 acctgaaaatgttatacggttcttcagcgatacggattgaatccagccc

4363 atagttacgtacatttgtattggcaaaacatgaatgtccaccgtctgtt |||||||||||||||||||||||||||||||||||||||||||||||||| 4401 atagttacgtacatttgtattggcaaaacatgaatgtccaccgtctgtt

4413 gcgaatgttgaataaaactccatttcaactttgtctgccaattgtccata ||||||||||||||||||||$.|||||||||||||||||||||||||||| \mid$ 4451 gcgaatgttgaataaactcaatttcaactttgtctgccaattgtccata

4463 gggttggttgttatatacctcggaatttgagaaaagatatccgatggcac |||||||||||||||||||||||||||||||||||||||||||||||||| $\mid$ 4501 gggttggttgttatatacctcggaatttgagaaaagatatccgatggcac

4513 atattaataaacagactttccaaacatgggtctgctgcag $\quad 4552$ || || || || || ||||||||||||||||||||||||||||| 4551 atattaataaacagactttccaaacatgggtetgctgcag 4591
3763

3800

3813

3850

3863

3900

3913

3950

3963

4000

4013

4050

4063

4100

4113

4150

4163

4200

4213

4250

4263

4300

4312

4350

4362

4400

4412

4450

4462

4500

4512

4550 
İzole edilen genin başlangıç kodu 1-3. bazlar, dur kodu ise 3589-3591. bazlar arasında bulunmaktadır. Ayrica, poliadenilazson sinyali ise 4053-4058. Baz arasında yer almaktadır. Yang et al. (1997)'nin bildirdiği dizide ise başlangıç kodu 1-3. bazlar, dur kodu 3552-3554. bazlar, poliadenilazson sinyali ise 4016-4021. bazlar arasında bulunmaktadır. Özellikle kültür soylarına ait gen havuzları çok farklı kaynak popülasyonlardan gelen bireylerin karılmasından oluşmaktadır. Bunun yanında 1slah çalışmaları ile kültür soyları istenilen karakter yönünde seçilmektedir (Carvalho, 1993). Dolayısıyla kültür popülasyonlarının genetik yapısı farklılıklar gösterebilmektedir. $\mathrm{Bu}$ çalışmada gözlenen farklılıkların bahsedilen populasyonlar arası varyasyondan kaynaklanabileceği düşünülmektedir.

GH geni üzerindeki ilgili putatif HRE'lere glukokortikoid, tiroid hormon ve retinoik asit gibi moleküllerin bağlanmasıyla genin hormonlar tarafindan düzenlenmesi sağlanmaktadır (Barlow et al., 1985; Slater et al., 1985). Tablo 1'de işaretlenmiş olan putatif HRE'ler (GRE, PRE, ARE, MRE, ERE, RXR, RAR, TR) daha önce rapor edilen çalışma (Yang et al., 1997) verileri ile uyum içerisindedir. Bu durum tespit edilen 54 nükletotit varyasyonun genin HRE'ler yoluyla düzenlenmesinde farklılık yaratmadığını düşündürmektedir.

$\mathrm{Bu}$ çalışmada Antalya İli'inde yetiştirilen gökkuşağı alabalığı popülasyonundan büyüme hormonu I geni izole ve karakterize edilmiştir. Genin intron bölgelerinde referans diziye kıyasla varyasyonların olduğu, bu varyasyonların genin ekzon bölgesinde olmamasından dolayı kodlanan aminoasit dizisi üzerinde herhangi bir etkisinin olmadığı görülmüştür. Ayrıca, intronların başlangıç ve bitiş dinükleotidleri klasik korunmuş uçbirleşme bölgeleri ile uyum içerisindedir. Bunun yanında yine referans diziyle kıyaslandığında incelenen hormon yanıt elementlerinin de varyasyonlardan etkilenmediği tespit edilmiştir.

\section{Sonuç}

Antalya ilinde üretilen gökkuşağı alabalığı çiftlik popülasyonundan izole ve karakterize edilen rtghI geninin daha önce rapor edilen referans dizi ile olan benzerlik ve farklılıkları ortaya konmuştur. Tespit edilen varyasyonlar intron bölgelerinde yer aldığından genin kodladığ yaratmamaktadır. Benzer şekilde intron bölgesinde yer alan değerlendirilen putatif HRE'ler de tespit edilen varyasyonlardan etkilenmemektedir. $\mathrm{Bu}$ çalışmada elde edilmiş olan veriler filogenetik ve gen aktarım çalışmalarında kullanılabilecek niteliktedir. Ayrıca, büyüme hormonu geninin ülkemizde kültürü yapılan tüm soylardaki benzerlik ve farklılıkları araştırılarak su ürünleri genetik 1slah programlarını destekleyecek verilerin oluşturulabileceği çalışmaların yapılmasına ihtiyaç vardır. Bu çalışmaların ışığında büyüme ve gelişme ile genetik varyasyon arasındaki ilişkiler araştırılabilir. Bu yolla verimli soylar tespit edilebilirse katma değeri daha yüksek üretim yapma olanağ doğacaktır.

\section{Teșekkür}

$\mathrm{Bu}$ çalışmada uygulanan prosedürler Akdeniz Üniversitesi Tıp Fakültesi Hayvan Bakım-Kullanım ve Hayvan Deneyleri Etik Kurulu tarafından değerlendirilmiş ve 2009.09.40 nolu karar ile etik olarak uygunluğu onaylanmıştır. Çalışma, Akdeniz Üniversitesi Bilimsel Araştırma Projeleri Koordinasyon Birimi tarafindan 2009.03.0121.012 no'lu proje ile desteklenen Mesut YILMAZ'a ait doktora tezinden üretilmiştir.

\section{Kaynakça}

Agellon, L.B. \& Chen, T.T. (1986). Rainbow trout growth. hormone: molecular cloning of cDNA and expression in Escherichia coli. DNA, 5(6), 463-471.

Agellon, L.B., Davies, S.L., Chen, T.T. \& Powers, D.A. (1988). Structure of a fish (Rainbow trout) growth hormone gene and its evolutionary 1mplications. Proceedings of the National Academy of Sciences of the United States of America, 85, 5136-5140.

Barlow, J. W., Voz, M. L., Eliard, P. H., Mathy-Harter, M., De Nayer, P., Economidis, I. V., Martial, J. A. \& Rousseau, G. G. (1986). Thyroid hormone receptors bind to defined regions of the growth hormone and placental lactogen genes. Proceedings of the National Academy of Sciences, 83(23), 9021-9025.

Ber, R., \& Daniel, V. (1993). Sequence analysis suggests a recent duplication of the growth hormone-enco ding gene in Tilapia nilotica. Gene, 125, 143-150.

Bhatia, S. \& Dahiya, R. (2015). Concepts and Techniques of Plant Tissue Culture Science: içinde Modern Applications of Plant Biotechnology in Pharmaceutical Sciences, Academic Press, California.

Björnsson, B.T. (1997). The biology of salmon growth hormone: from daylight to dominance. Fish Physiology and Biochemistry, 17: 9-24.

Brody, J.R. \& Kern, S.E. (2004). History and principles of conductive media for standard DNA electrophoresis. Analytical Biochemistry, 333(1), 1-13.

Carvalho, G. R. (1993). Evolutionary aspects of fish distribution: genetic variability and adaptation. Journal of Fish Biology, 43(sa), 53-73.

Clark, D. P. \& Pazdernik, N. J. (2013). DNA Sequencing: içinde Molecular Biology (Second Edition), Academic Press, California.

Dieffenbach, C.W., Lowe, T.M.J. \& Dveksler, G.S. (1995). General concepts for PCR primer design 1n: PCR Primer, A Laboratory Manual, Dieffenbach CW, Dveksler GS Ed., Cold Spring Harbor Laboratory Press, New York.

Filiz, E. \& Koç, İ. (2011). Bitki Biyoteknolojisinde Moleküler Markörler. GOÜ, Ziraat Fakültesi Dergisi, 28(2), 207-214.

Freeland, J. R. (2008). Ekologia Molekularna. Wydawnictwo Naukowe PWN, Warszawa.

Gerstein, M.B., Bruce, C., Rozowsky, J.S., Zheng, D., Du, J., Korbel, J.O., Emanuelsson, O., Zhang, Z.D., Weissman, S. \& Snyder, M. (2007). What is a gene, post-ENCODE? History and updated definition. Genome Research, 17 (6), 669-681.

Hall, T.A. (1999). BioEdit: a user-friendly biological sequence alignment editor and analysis program for Windows 95/98/NT. Nucleic Acids Symposium Series, 41, 95-98.

Hüttenhofer, A., Schattner, P. \& Polacek, N. (2005). Non-coding RNAs: hope or hype?. Trends Genet., 21 (5), 289-97.

Johansen, B., Johnsen, O.C., \&Valla, S. (1989). The complete nucleotide sequence of the growth hormone gene from Atlantic salmon (Salmo salar). Gene, 77, 317-324.

Kalendar, R., Lee, D. \& Schulman, A.H. (2009). Fast PCR Software for PCR Primer and Probe Design and Repeat Search. Genes, Genomes and Genomics, 3(1), 1-14. 
Kaplan, S. L. (1999) Hormonal regulation of growth and metabolic effects of growth hormone. In: Kostyo JL, Goodman HM (Eds) Handbook of Physiology. The endocrine system, Vol V: Hormonal control of growth. Oxford University Press, New York.

Kennedy, S. \& Oswald, N. (2011). PCR Troubleshooting and Optimization: The Essential Guide. Caister Academic Press, Norfolk, UK.

Klug, W.S., Cummings, M.R., Palladino, M. A. \& Spencer, C. A. (2018). Genetik Kavramlar (11. Bask1). Palme Yayıncılık, Ankara.

Kocher, T.D. \& Carleton, K.L. (1997). Base Substitution in Fish Mitochondrial DNA: Patterns and Rates: içinde Molecular Systematics of Fishes, pp 13-24. Academic Press, California.

Male, R., Nerlaand, A.H., Lorens, J.B., Telle, W., Lossius, I., \& Totland, G.K. (1992). The complete nucleotide sequence of the Atlantic salmon growth hormone I gene. Biochem. Biophys. Acta, 1130, 345-348.

Marins, L. F., Levy, J. A., Folch, J. M. \& Sanchez, A. (2003). A growth hormone-based phylogenetic analysis of euteleostean fishes including a representative species of the Atheriniformes Order, Odontesthes argentinensis. Genetics and Molecular Biology, 26 (3), 295-300.

Mori, T., Deguchi, F. \& Ueno, K. (2001). Differential Expression of GH1 and GH2 Genes by Competitive RT-PCR in Rainbow Trout Pituitary. General and Comprative Endocrinology, 123, 137-143.

Mount, S.M. (1982). A catalogue of splice junction sequences. Nucleic Acids Research, 10, 459-472.

Nelson, D. L. \& Cox, M. M. (2017). Lehninger Principles of Biochemistry (7th Ed.). W.H. Freeman and Company, New York.

Nicoll, C. S., Rodgers, B. D. \& Kelly, K. M. (1999) Hormonal regulation of growth and development of nonmammalian vertebrates. In: Kotsyo JL, Goodman HM (Eds) Handbook of physiology. The endocrine system, Vol V, Hormonal control of growth. Oxford University Press, NY.

Özcan-Gökçek, E., \& Işık, R. (2020). Associations between genetic variants of the insulin-like growth factor I (IGF-I) gene and growth traits in European sea bass (Dicentrarchus labrax, L.). Fish Physiology and Biochemistry, 46, 11311138.

Rajesh, R. \& Majumdar, K. C. (2007). A comparative account of the structure of the growth hormone encoding gene and genetic interrelationship in six species of the genus Labeo. Fish Physiol Biochem, 33, 311-333.

Rothan, H. A., Ser Huy, T., \& Mohamed, Z. (2014). Effect of Codon Optimisation on the Production of Recombinant Fish Growth Hormone inPichia pastoris. The Scientific World Journal, 1-6.

Sekine, S., Mizukami, T., Nishi, T., Kuwana, Y., Saito, A., Sato, M., Itoh, S. \& Kawauchi, H. (1985). Cloning and expression of cDNA for salmon growth hormone in Escherichia coli. Proceedings of the National Academy of Sciences of the United States of America, 82, 4306-4310.

Slater, E. P., Rabenau, O., Karin, M., Baxter, J. D., \& Beato, M. (1985). Glucocorticoid receptor binding and activation of a heterologous promoter by dexamethasone by the first intron of the human growth hormone gene. Molecular and Cellular Biology, 5(11), 2984-2992.

Thompson, J. D., Higgins, D. G., \& Gibson, T. J. (1994). CLUSTAL W: improving the sensitivity of progressive multiple sequence alignment through sequence weighting, position-specific gap penalties and weight matrix choice. Nucleic Acids Research, 22(22), 4673-4680.

Wagner, G.F., Fargher, R.C., Brown, J.C. \& Mckeown, B.A. (1985). Further chacterization of growth hormone from chum salmon (Oncorhynchus keta). General and Comparative Endocrinology, 60, 27-34.

Xia, J., Xia, K. \& Jiang, S. (2008). Complete mitochondrial DNA sequence of the yellowfin seabream Acanthopagrus latus and a genomic comparison among closely related sparid species. Mitochondrial DNA, 19(4), 385-393.

Yang, B., Chan, K., Lin C. \& Chen, T.T. (1997).

Characterization of rainbow trout (Oncorhynchus mykiss) growth hormone 1 gene and the promoter region of growth hormone 2 gene. Archives of Biochemistry Biophysic, 340, 359-368.

Yılmaz, M. 2012. Dă̆ Alası (Salmo trutta macrostıgma Dumeril, 1858) için $\beta$-aktin Promotör-rtghı İfade Vektörünün Hazırlanmasi. Doktora Tezi, Akdeniz Üniversitesi, Fen Bilimleri Enstitüsü, Antalya.

Yowe, D.L., \& Epping, R.J. (1995). Cloning of the barramundi growth hormone-encod ing gene: a comparative analysis of higher and lower vertebrate GH genes. Gene, 162, 255-259. 